\title{
Characterization and in vitro and in vivo evaluation of cross-linked chitosan films as implant for controlled release of citalopram
}

\author{
PATIT P KUNDU*, SANTOSH KUMAR JINDAL and MANISH GOSWAMI ${ }^{\dagger}$ \\ Department of Polymer Science and Technology, University of Calcutta, Kolkata 700 009, India \\ ${ }^{\dagger}$ Akal College of Pharmacy and Technical Education, Mastuana, Sangrur 148 001, India
}

MS received 21 November 2008; revised 30 June 2012

\begin{abstract}
The aim of the present study is to develop cross-linked chitosan $(\mathrm{CH})$ films that can release drug over an extended period of time and that too in a controlled manner. A solution of different percentages of $\mathrm{CH}$, is prepared in $1 \%$ lactic acid, followed by addition of citalopram (CTP) and then reacted with increasing amounts of glutaraldehyde (GL) to obtain films with different cross-linking densities. Prepared films are characterized for their physical and mechanical properties. The films are then subjected to in vitro drug release studies using pH 7.4 phosphate buffer saline (PBS) as dissolution medium and cumulative amount of drug released is calculated. Kinetic analysis of drug release is performed using Power law model and Higuchi's model. With increase in concentration of CH, water absorption capacity and mechanical strength are increased; whereas, water vapour permeability and elasticity of the films are decreased. The effect of cross-linking agent, GL, is such that with an increase in the amount of GL, water vapour permeability, water absorption capacity and elasticity of the films are decreased; whereas, mechanical strength increased to some extent and then decreased. In vitro release studies indicate that films containing $3 \% \mathrm{CH}$, cross-linked with 2-3\% GL and films containing 4\% CH, cross-linked 1\% GL are able to sustain the drug release for a prolonged time along with releasing almost complete drug in a desired period. Out of these batches, films containing 3\% CH, cross-linked with 2-3\% GL are having sufficient strength, water vapour permeation, water absorption capacity and elongation at break for implantation purpose. The in vitro degradation studies and histopathological studies were carried out with a sample film (batch $\mathrm{C} 3$ as in table 1) in rabbit model. In vitro degradation study indicates that the films maintained their integrity for desired implantation. The histopathological studies under optical microscope indicates that on implanting, there is no evidence of any inflammation, any foreign body granuloma or any necrosis or hemorrhage. Tissue configuration remains unaltered after 30 days of implantation. So, it can be suggested that cross-linked $\mathrm{CH}$ films of above said composition can be used as implant for long term application in depression and related disorders.
\end{abstract}

Keywords. Chitosan film; citalopram; physical properties; mechanical properties; in vitro drug release.

\section{Introduction}

Depression and related disorders like schizophrenia, obsessive compulsive disorders, panic attacks and other anxiety disorders are leading to a large number of mortalities or other health hazards. A large variety of drugs including tricyclic/tetracyclic antidepressants are in use in the last decade to treat depression and related disorders (Rickels and Schweizer 1990; Preskorn 1995). Since early 1960s, imipramine, amitriptyline, their $N$-demethyl derivatives, and other similar compounds are the first successful antidepressants. Because of their structure, these agents often are referred to as the 'tricyclic' antidepressants (Frazer 1997). Their efficacy in alleviating major depression is well established and they are also useful in a number of other psychiatric disorders. Just prior to the discovery of the antidepressant properties of imipramine in the late 1950s, the ability

\footnotetext{
*Author for correspondence (ppk923@yahoo.com)
}

of 'Monoamine Oxidase (MAO) Inhibitors' to cause mania is noted. During the early 1960s, both types of agents were studied extensively in the treatment of clinical depression. Earlier, MAO inhibitors appeared to be limited in efficacy at the doses used and presented both toxic risks and potentially dangerous interaction with other agents, thus limiting their acceptance in favour of the tricyclic agents (Frazer 1997).

After decades of limited progress, a series of innovative antidepressants has emerged. Most of them like citalopram, fluoxetine, fluvoxamine, venlafaxine, paroxetine and sertraline are inhibitors of the active reuptake of serotonin (5-hydroxytryptamine, 5-HT) into nerve terminals. Others including bupropion, nefazodone and mirtazapine have a less well defined neuropharmacology and can be considered 'atypical'. Whereas the efficacy of the newer agents is not superior to that of the older agents, their relative safety and tolerability has led to their rapid acceptance as the most commonly prescribed antidepressants. So, in the modern pharmacotherapy, drugs from the category of the "Selective Serotonin Reuptake Inhibitors (SSRIs)" are considered as the first line therapy to treat depression and related disorders 
because of their documented efficacy and rather advantageous side effect profile as compared to tricyclic/tetracyclic antidepressants (Andrews and Nemeroff 1994).

Chitosan is a biopolymer, obtained by $N$-deacetylation of chitin, which is the second most abundant polysaccharide on the earth after cellulose. It is commercially available from a stable renewable source, i.e. shellfish waste (shrimp and crab shells) of the sea-food industry (Arvanitoyannis et al 1998; Crini 2006). It has been reported that chitosan is a potentially useful pharmaceutical material owing to its good biocompatibility and low toxicity (Thomas and Sharma 1990). For these reasons, chitosan has been used in many applications in the formulations employed in drug delivery (Miyazaki et al 1981; Gupta and Kumar 2000). Since chitosan can be hydrolyzed by lysozyme, it is also one of the biodegradable polymers in nature. The degradation rate of chitosan can be controlled by changing its polymer composition (i.e. the copolymerization ratio of glucosamine to $N$-acetylglucosamine or the length of acyl side-chain on $N$-acetylglucosamine) and/or its molecular weight (David et al 1992; Tomihata and Ikada 1997). Furthermore, the degraded products of chitosan are nontoxic, nonimmunogenic and noncarcinogenic (Muzzarelli 1993). Chitosan membranes have been explored in many cases, such as enzyme immobilization and cationic specimen transportation (Miao and Tan 2000; Adriano et al 2005), protein separation and concentration (Bayramocglu et al 2003; Vieira and Beppu 2006), controlled ingredientrelease (Blanco et al 2000; Mi et al 2001; Wang et al 2005) and environmental applications (Ngah et al 2002; Beppu et al 2004; Guibal 2004; Vieira and Beppu 2005). Chitosan is a well known filmogenic material. Further treatments are usually done in order to turn chitosan more bacteriostatic and to improve chemical and mechanical resistance. A study has explored a very interesting route to modify chitosan functional groups, mostly composed of amino groups, using glutaraldehyde (Vieira and Beppu 2005). For most of the drug delivery applications, the chitosan should be cross-linked due to its hydrophilic properties. Studies have shown that the glutaraldehyde cross-linked chitosan microspheres have a long-acting biodegradable ability suitable for controlleddelivery of many drugs (Jameela et al 1994, 1998; Jameela and Jayakrishnan 1995; Genta et al 1998). Cross-linking of chitosan using glutaraldehyde makes it less susceptible to degradation by lysozyme. Additionally, the glutaraldehyde cross-linked chitosan microspheres have been shown to have the ability for long-acting delivery of drugs (Jameela et al 1994; Jameela and Jayakrishnan 1995). In a study, heterogeneous cross-linking with glutaraldehyde have shown to produce more hydrophobic structures in chitosan membranes (Beppu et al 2007).

The development of a new, safe, specific and effective dosage form is an integrated time and cost intensive venture. Pharmaceutical manufacturers have shown a renewed interest in improving the already existing dosage forms and developing more sophisticated drug delivery systems employing the principles of modified drug administration and release technology. Implantable drug delivery is one of such technologies, by which a pharmaceutical formulation having better absorption, better site specificity, prolonged release, reduced dosing frequency and low toxicity have been designed.

Implants offer several advantages over other drug delivery systems such as (i) slow drug absorption for prolonged time period, (ii) by-passing first pass metabolism, (iii) reduced gastro-intestinal side effects, (iv) reduced dosing frequency and increased patient compliance, (v) small dose is sufficient to elicit the action, (vi) better drug plasma concentration profile, (vii) release the drug in a rate controlled manner which leads to enhanced effectiveness and reduction in side effects, (viii) increased biological activity due to better site specificity and (ix) readily retrievable by medical personnel to terminate medication.

The main advantage for which we considered to choose implantable drug delivery for the treatment of depression was patient compliance. Often in the case of therapy for depression and related disorders, patient does not take drug regularly or stops in between, whenever the mood is altered or elevated. In India, due to trend of Ayurvedic treatment and misconceptions of depression relating with madness, many patients try to avoid antidepressants. Implants by virtue of their mode of delivery i.e. non-visibility of formulation ingestion, overcome this problem.

Sezer et al (2007) reported that the chitosan film containing fucoidan can be used as a wound dressing material for healing of dermal burn. Thus, chitosan films can be used in the dermal region for the release of citalopram, an antidepressant.

The present study is aimed at developing modified drug delivery system containing antidepressant drug, citalopram. Patient compliance is the major factor, especially in the case of depressed patients, for which we have considered to investigate a modified drug delivery system based on chitosan that can release an antidepressant over an extended period of time (21-30 days) and that too in a controlled manner.

\section{Materials and methods}

\subsection{Materials}

Citalopram was obtained as a gift sample from Ind-Swift Ltd., Parwanoo (H.P.), India. Chitosan (purified, degree of deacetylation, 85\%) was obtained from Indian Sea Foods, Cochin, India. All other chemicals and reagents used were of analytical grade.

\subsection{Preparation of citalopram-chitosan films}

Chitosan $(\mathrm{CH})$ was dispersed in $1 \%$ lactic acid solution and heated to $100^{\circ} \mathrm{C}$. After adjusting the temperature to $30^{\circ} \mathrm{C}$, citalopram (CTP) (3\%) was added into chitosan solution, stirred thoroughly and followed by the addition of propylene glycol $(2.5 \%)$ as a plasticizer. To this mixture, glutaraldehyde (GL) (stock solution, 25\% w/v) was quickly added while 
stirring. Immediately, the resulting mixture was transferred into a petri dish and kept in a hot air oven at $40^{\circ} \mathrm{C}$ for drying. Amount of $\mathrm{CH}$ and GL added were varied in different batches as shown in table 1. After drying, the films were peeled off and stored in an air tight container at room temperature until further investigation. Codes $A, B, C$ and $D$ represent films prepared with 1, 2, 3 and $4 \% \mathrm{CH}$, respectively. Batches $A 1-A 4, B 1-B 4, C 1-C 4$ and $D 1-D 4$ represent films cross-linked with $1-4 \%$ concentration of GL in all cases.

\subsection{Cross-link density measurement}

The cross-link density of the films was quantified by adsorption measurements of the negatively charged dye, eosin, from a hydroalcoholic solution (Gliko-Kabir et al 2000). In different studies, about $0.2 \mathrm{~g}$ of each film was incubated in $2 \mathrm{ml}$ of $0.05 \mathrm{mg}$ of eosin in 1:1 ethanol: water solution for $3 \mathrm{~h}$ at room temperature. The films were then removed and the eosin concentration in the incubation medium was measured spectrophotometrically $(520 \mathrm{~nm})$, using a six-point calibration curve. The films were then rinsed with water, dried in acetone (48 h) and weighed. The amount of eosin adsorbed, which was calculated from the initial and final concentrations of eosin in the bathing solution, was normalized to the dry weight of each film.

\subsection{Water vapour permeability of films}

The prepared films were tied onto the mouth of borosilicate glass bottles (capacity, $30 \mathrm{ml}$; diameter of top hole, $22 \mathrm{~mm}$ ) filled with anhydrous calcium chloride. All the bottles were put into a desiccator containing saturated sodium chloride solution ( $65 \pm 5 \%$ relative humidity) and stored in an oven maintained at $25 \pm 1^{\circ} \mathrm{C}$. The average area available for vapour permeation was $14.5 \mathrm{~cm}^{2}$. After 28 days, the containers were weighed three times to test the reproducibility (RemunanLopez and Bodmeier 1997).

\subsection{Water absorption capacity of films}

The films were suspended in glass bottles containing $25 \mathrm{ml}$ of phosphate-buffer saline (PBS) $\mathrm{pH} 7.4$ and incubated in a shaking incubator at $37^{\circ} \mathrm{C}$ and $50 \mathrm{rpm}$. After a time interval of $12 \mathrm{~h}$, the films were taken out, excess water was removed carefully with filter paper and then weighed immediately (Shu et al 2001). Measurements were performed three times to test the reproducibility.

\subsection{Mechanical properties}

The mechanical properties of the films were measured using a texture analyzer (TA. XT Plus, Texture Technologies, USA) equipped with a $5 \mathrm{~kg}$ load cell. A film strip (dimension, $2 \times 2 \mathrm{~cm}$ ) was held between 2 clamps and pulled by the top clamp at a rate of $0.5 \mathrm{~mm} / \mathrm{s}$. The force and elongation were measured when the film broke off (Kalapathy et al 2000). The values were the average of three experiments. The tensile strength and elongation at break were calculated by (1) and (2).

$$
\mathrm{TS}=F / A^{2},
$$

where TS is the tensile strength $\left(\mathrm{N} / \mathrm{mm}^{2}\right), F$ the breaking force $(N)$ and $A$ the cross-sectional area of sample $\left(\mathrm{mm}^{2}\right)$.

$$
\mathrm{EB}=\left(L-L_{0} / L_{0}\right) \times 100,
$$

\begin{tabular}{|c|c|c|c|c|c|c|}
\hline Batch no. & $\begin{array}{l}\text { Chitosan } \\
(\% \mathrm{w} / \mathrm{v})\end{array}$ & $\begin{array}{c}\text { Glutaraldehyde } \\
(\% \mathrm{v} / \mathrm{v})\end{array}$ & $\begin{array}{c}\text { Water vapour } \\
\text { permeability\# } \\
(g \pm \text { S.D. })\end{array}$ & $\begin{array}{l}\text { Water absorption } \\
\text { capacity } \\
\text { ( } g \pm \text { S.D. })\end{array}$ & $\begin{array}{c}\text { Tensile } \\
\text { strength } \\
\text { (N土 S.D.) }\end{array}$ & $\begin{array}{c}\text { Film } \\
\text { elongation } \\
(\% \pm \text { S.D. })\end{array}$ \\
\hline$A 1$ & $1 \cdot 0$ & $1 \cdot 0$ & $15 \cdot 4 \pm 0.5$ & $0.82 \pm 0.03$ & $16 \cdot 6 \pm 0 \cdot 8$ & $22 \cdot 4 \pm 0 \cdot 4$ \\
\hline$A 2$ & $1 \cdot 0$ & $2 \cdot 0$ & $14 \cdot 7 \pm 0 \cdot 2$ & $0 \cdot 80 \pm 0 \cdot 12$ & $17 \cdot 2 \pm 0 \cdot 7$ & $21 \cdot 0 \pm 0 \cdot 6$ \\
\hline$A 3$ & $1 \cdot 0$ & $3 \cdot 0$ & $14 \cdot 2 \pm 0 \cdot 2$ & $0.76 \pm 0.06$ & $17 \cdot 0 \pm 1 \cdot 2$ & $20 \cdot 7 \pm 0 \cdot 1$ \\
\hline$A 4$ & $1 \cdot 0$ & $4 \cdot 0$ & $14 \cdot 0 \pm 0 \cdot 4$ & $0.73 \pm 0.02$ & $16 \cdot 8 \pm 1 \cdot 2$ & $20 \cdot 4 \pm 0 \cdot 2$ \\
\hline$B 1$ & $2 \cdot 0$ & $1 \cdot 0$ & $12 \cdot 9 \pm 0.4$ & $1.65 \pm 0.04$ & $38 \cdot 1 \pm 2 \cdot 3$ & $17 \cdot 6 \pm 0 \cdot 6$ \\
\hline$B 2$ & $2 \cdot 0$ & $2 \cdot 0$ & $12 \cdot 2 \pm 0 \cdot 7$ & $1.58 \pm 0.06$ & $45 \cdot 6 \pm 1 \cdot 4$ & $16 \cdot 8 \pm 0 \cdot 5$ \\
\hline$B 3$ & $2 \cdot 0$ & $3 \cdot 0$ & $11 \cdot 6 \pm 0.4$ & $1.50 \pm 0.04$ & $42 \cdot 9 \pm 0 \cdot 8$ & $16 \cdot 3 \pm 0 \cdot 5$ \\
\hline$B 4$ & $2 \cdot 0$ & $4 \cdot 0$ & $11 \cdot 2 \pm 0 \cdot 8$ & $1.47 \pm 0.04$ & $39 \cdot 5 \pm 1 \cdot 3$ & $15 \cdot 7 \pm 0 \cdot 4$ \\
\hline$C 1$ & $3 \cdot 0$ & $1 \cdot 0$ & $9 \cdot 6 \pm 0 \cdot 5$ & $1.84 \pm 0.03$ & $46 \cdot 3 \pm 1 \cdot 1$ & $13 \cdot 8 \pm 0 \cdot 6$ \\
\hline$C 2$ & $3 \cdot 0$ & $2 \cdot 0$ & $9.4 \pm 0.2$ & $1.77 \pm 0.01$ & $47 \cdot 1 \pm 2 \cdot 7$ & $13 \cdot 6 \pm 0 \cdot 2$ \\
\hline$C 3$ & $3 \cdot 0$ & $3 \cdot 0$ & $8 \cdot 8 \pm 0.2$ & $1.75 \pm 0.13$ & $46 \cdot 8 \pm 1 \cdot 5$ & $12 \cdot 5 \pm 0.6$ \\
\hline$C 4$ & $3 \cdot 0$ & $4 \cdot 0$ & $8 \cdot 8 \pm 0.3$ & $1.71 \pm 0.04$ & $45 \cdot 6 \pm 0 \cdot 9$ & $11 \cdot 7 \pm 0 \cdot 1$ \\
\hline$D 1$ & $4 \cdot 0$ & $1 \cdot 0$ & $5 \cdot 1 \pm 0 \cdot 3$ & $1.95 \pm 0.07$ & $48 \cdot 2 \pm 0 \cdot 8$ & $6.8 \pm 0.4$ \\
\hline D2 & $4 \cdot 0$ & $2 \cdot 0$ & $4 \cdot 6 \pm 0.2$ & $1.91 \pm 0.06$ & $48 \cdot 9 \pm 1 \cdot 3$ & $6 \cdot 3 \pm 0.4$ \\
\hline$D 3$ & $4 \cdot 0$ & $3 \cdot 0$ & $4 \cdot 2 \pm 0 \cdot 1$ & $1.88 \pm 0.04$ & $47 \cdot 5 \pm 1 \cdot 0$ & $5.8 \pm 0.7$ \\
\hline$D 4$ & $4 \cdot 0$ & $4 \cdot 0$ & $3 \cdot 4 \pm 0 \cdot 2$ & $1.85 \pm 0.06$ & $47 \cdot 1 \pm 1 \cdot 7$ & $5 \cdot 1 \pm 0 \cdot 5$ \\
\hline
\end{tabular}

Table 1. Designation, composition, physical and mechanical properties of cross-linked chitosan films. 
where EB is the elongation at break (\%), $L$ the initial length $(\mathrm{mm})$ and $L_{0}$ the extended length $(\mathrm{mm})$.

\subsection{In vitro drug release studies}

The drug release from cross-linked chitosan films was performed under the same conditions as described in the water absorption studies. At appropriate time intervals, the solutions were withdrawn and the content of the model drug were determined spectrophotometrically at a wavelength of $238 \mathrm{~nm}$. An equal volume of the same dissolution medium was added back to maintain a constant volume. Maximum period of study was thirty days. In vitro release studies were performed for all the batches in PBS (pH 7.4) as in our future studies, selected formulations were implanted in intradermal region having $\mathrm{pH} 7 \cdot 4$.

\subsection{Kinetic analysis of drug release}

In order to have an insight into the mechanism of drug release behaviour of the cross-linked films, the power law model and Higuchi's model were fitted into the kinetic data of drug release. Power law model (Korsmeyer et al 1983) (known as Korsmeyer-Peppas equation) can be expressed as:

$$
M_{\mathrm{t}} / M_{\infty}=k t^{n} .
$$

According to Higuchi's (1963) model, an inert matrix should provide a sustained drug release over a reasonable period of time and yield a reproducible straight line when the fraction of drug released is plotted vs the square root of time. Higuchi's model can be expressed as:

$$
M_{\mathrm{t}} / M_{\infty}=k t^{1 / 2},
$$

$M_{\mathrm{t}}$ is the amount of drug released in time ' $t$ ', $M_{\infty}$ corresponds to the total amount of drug released after an infinite time, $k$ a constant related to the structural and geometric properties of the drug release system and $n$ the diffusional exponent. The numerical value of $n$ provides information about the mass transport mechanism for release studies. When $n<0 \cdot 5$, the solvent diffuses through and the drug is released from the polymeric matrix with a quasiFickian diffusion mechanism. An anomalous, non-Fickian drug diffusion occurs when the value of $n$ lies between 0.5 and 1 . If $n \geq 1$, a non-Fickian, case II or zero-order release kinetics can be observed.

\subsection{In vivo degradation study}

Batch $C 3$ films (as in table 1) selected on the basis of in vitro studies were subjected to in vivo studies in rabbits. Three male New Zealand albino rabbits weighing around $1.5-2.5 \mathrm{~kg}$ were used as animal models in these studies. The animals were caged separately and housed under environmentally controlled conditions like $37{ }^{\circ} \mathrm{C}$ temperature and
$12 \mathrm{~h}$ lighting cycle. The animals were fed with a standard diet available commercially and had access to water.

Cross-linked chitosan films (Batch $C 3$ ) were implanted subcutaneously in the thoracic region of the rabbits. On the day of implantation, the thoracic region was shaved with sterile blade and cleaned by alcohol swab. Implantation was conducted through incision in the thoracic region under local anesthetic condition with xylocaine injection. One sterile implant sample $\left(4 \mathrm{~cm}^{2}\right.$ film) was inserted at the subcutaneous site and the stitching was done using sterile needle and nylon thread. Following surgery, the rabbits were supervised until complete recovery from anesthesia and then normal diet was resumed. After 30 days, the specimen was retrieved, rinsed in water, dried and weighed $\left(W_{\text {rem }}\right)$. The extent of film degradation, in percent of initial amount (\% remained), was calculated using the following equation:

$$
\% \text { Remained }=\left(W_{\text {rem }} / W_{0}\right) \times 100,
$$

where $W_{\text {rem }}$ is the dry weight of the film debris retrieved at the end of the implantation study and $W_{0}$ the initial dry weight of the film.

\subsection{Histopathology study}

The word histopathology is derived from two Greek wordshistos (tissue) and pathos (suffering). Histopathology is the examination of tissues from the body under a microscope to spot the signs and characteristics of changes in tissue configuration or disease. A histopathology report describes the tissue that has been sent for examination and what its features are under the microscope. Occasionally a histopathology report is also called a biopsy report.

Histopathology study on rabbit was conducted in parallel to in vivo degradation study to determine formulation compatibility. On the day of implantation of film, when incision was given in the thoracic region of the rabbit, a tissue sample was taken from subcutaneous site (the same site where implant was inserted afterwards) with no. 5 skin punch biopsy stainless steel forceps and sent for histopathology study.

After 30 days, when the implant specimen was retrieved, one more tissue biopsy sample was taken from the same site and sent for histopathology study.

\section{Results and discussion}

Smooth, clean, firm and tough films of $\sim 1 \mathrm{~mm}$ thickness are obtained using different concentrations of $\mathrm{CH}$ (containing CTP) and cross-linked with varied amounts of GL.

\subsection{Cross-link density measurement}

In batches containing $1-4 \% \mathrm{CH}$, increasing amounts of GL are used to prepare a series of $\mathrm{CH}$ films with different crosslinking densities that are characterized by eosin adsorption. 
The more crosslinked chitosan film has less active site (i.e amine group) for adsorption of eosin. In figure 1, the concentration of eosin adsorbed is plotted against the concentration of glutaraldehyde used for crosslinking of the films. From the figure, it is observed that the amount of eosin adsorbed is inversely proportional to the relative amount of GL used for cross-linking. The study indicates that in case of films prepared with $1 \%$ and $2 \% \mathrm{CH}$, the reaction reached its end-point at $2 \%$ GL concentration. In case of films containing $3 \%$ and $4 \% \mathrm{CH}$, eosin adsorption decreases with an increase in GL concentration from 1 to $4 \%$. This may be due to the reason that in case of films containing $1 \%$ and $2 \% \mathrm{CH}$, the binding sites $\left(-\mathrm{NH}_{2}\right)$ get saturated with $2 \%$ GL. Therefore, further increments in GL do not show any significant effect. On the other hand, in case of $3 \%$ and $4 \% \mathrm{CH}$, sufficient $-\mathrm{NH}_{2}$ groups are available for binding with GL up to its $4 \%$ concentration, thereby increasing cross-link density with an increase in GL concentration.

\subsection{Physical and mechanical properties of cross-linked chitosan films}

As shown in table 1 , water vapour permeability values of films vary between 3.4 and $15.4 \mathrm{~g}$, water absorption capacity from 0.73 to $1.95 \mathrm{~g}$, tensile strength values from $16 \cdot 6$ to $48.9 \mathrm{~N}$ and elongation at break values range from $5 \cdot 1$ to $22.4 \%$. Water vapour permeability of the films is significantly decreased with an increase in concentration of $\mathrm{CH}$ in the formulation. This is due to the reason that as the concentration of $\mathrm{CH}$ increases (for films of same thickness), void volume of the film decreases, toughness increases and so, the permeation of water vapours through the film decreases. Besides this, the amount of cross-linking agent GL is also found to be an effective parameter for controlling the permeability. For batches with the same concentration of $\mathrm{CH}$, the water vapour permeability decreases with an increase in GL concentration. This is due to the reason that as the concentration of cross-linking agent increases (for films of the same $\mathrm{CH}$ concentration), the films become more firm, rigid and tough. Thus, the water vapour permeability through the films decreases. Water absorption capacity is affected by the same parameters that had an effect on the water vapour

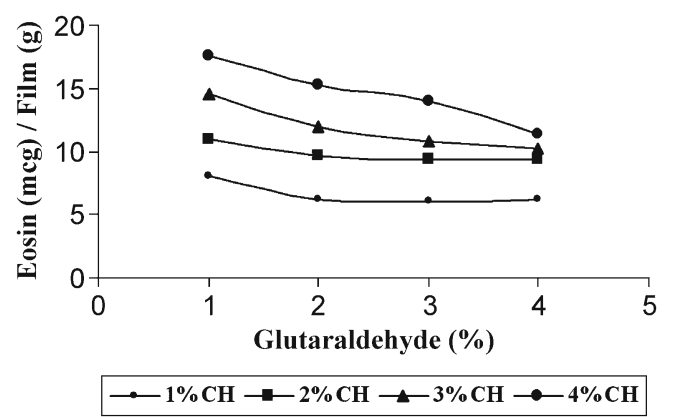

Figure 1. Variation of eosin absorption with variation in crosslink density in terms of variable contents of glutaraldehyde in films. permeability. Study indicates that water absorption capacity increases with increase in the concentration of chitosan in the film and decreases with increase in the amount of GL. This may be due to the reason that as the concentration of $\mathrm{CH}$ increases, toughness of the film increases, film becomes tortuous and the path of solvent through the film becomes nonlinear. Thus, less solvent oozes out through the film. Whereas, with increase in concentration of cross-linker, film becomes more firm, rigid and tough, which leads to decrease in water absorption capacity. Findings of this study demonstrate that with an increase in the concentration of $\mathrm{CH}$, toughness of the film increases and so, the tensile strength increases, but on the other hand, elongation decreases. The effect of amount of GL on tensile strength of the films is such that as the amount of GL increases from $1 \%$ to $2 \%$, tensile strength increases; but with still higher addition of GL (3 and $4 \%$ ), tensile strength of the films decreases. From this study, it may be suggested that up to some extent, GL increases the tensile strength but afterwards it increases the brittleness of the film and so the strength decreases. Also the study indicates that elongation of the films decreases with increase in the amount of GL. This is due to the reason that with increase in concentration of GL, toughness and rigidity of the film increases and so, the elongation decreases.

\subsection{In vitro drug release studies}

In vitro release studies are performed in $\mathrm{PBS} \mathrm{pH} 7.4$ for all the batches and the percent cumulative release of the CTP over a period of time (in days) is shown graphically in figure 2. The study indicates that batches $A 1, A 2, A 3$ and $A 4$ films release about $95-97 \%$ of the drug in $6,9,9$ and 10 days, respectively. In case of batches $B 1, B 2, B 3$ and $B 4$ films, about $95-97 \%$ of the drug is released in 11,15 , 17 and 17 days, respectively. In case of batches $C 1, C 2$ and $C 3$ films, about 95-96\% of the drug is released in 16, 21 and 27 days, respectively. In case of batch $C 4$ film, $84.15 \%$ drug is released in 30 days. In case of batch $D 1$ film, $94.65 \%$ of drug is released in 23 days. In batches D2, D3 and D4 films, the drug release in 30 days is $80.47,68.12$ and $54.23 \%$, respectively. As per findings of the in vitro drug release studies, batches $A 1, A 2, A 3$ and $A 4$ films are not able to sustain the drug release for a prolonged time. The effect of cross-linking agent in these batches is such that an increase in GL concentration from 1 to $2 \%$ can prolong the drug release, but further increments of GL do not show any significant effect. Batches $B 1, B 2, B 3$ and $B 4$ films can sustain the drug release for somewhat longer time, but still not desirable. In these batches, the drug release time increases with increasing GL concentration from 1 to $3 \%$. Further increments of GL do not show any significant effect. Films of batches $C 1, C 2, C 3$ and $C 4$ can sustain the drug release for a prolonged time and as the concentration of GL increases from 1 to $4 \%$, drug release time increases. In batches $D 2$, $D 3$ and $D 4$ films, the drug release is sustained to that extent that the nearly complete drug release $(>94 \%)$ is not obtained 
even after desired thirty days. In batches $D 1$ to $D 4$, as the concentration of GL increases from 1 to $4 \%$, drug release time increases. For films containing the same concentration of $\mathrm{CH}$ and cross-linked with varying concentrations of GL and vice versa; with increase in concentration of GL, the diffusion of drug through the films decreases and thereby, the release rate of drug decreases. So, in vitro drug release studies indicate that batches $C 2, C 3$ and $D 1$ films can sustain the drug release for a prolonged time along with releasing almost complete drug in desired time period. If we compare physical and mechanical properties of batches $C 2, C 3$ and $D 1$ films, it can be seen that batches $C 2$ and $C 3$ films are having good tensile strength and sufficient water vapour permeability, water absorption capacity and elongation at break values. Batch $D 1$ films are having good tensile strength and water absorption capacity, but the water permeation and elongation at break value is very less. So, it can be concluded that batches $C 2$ and $C 3$ films are having desirable physical and
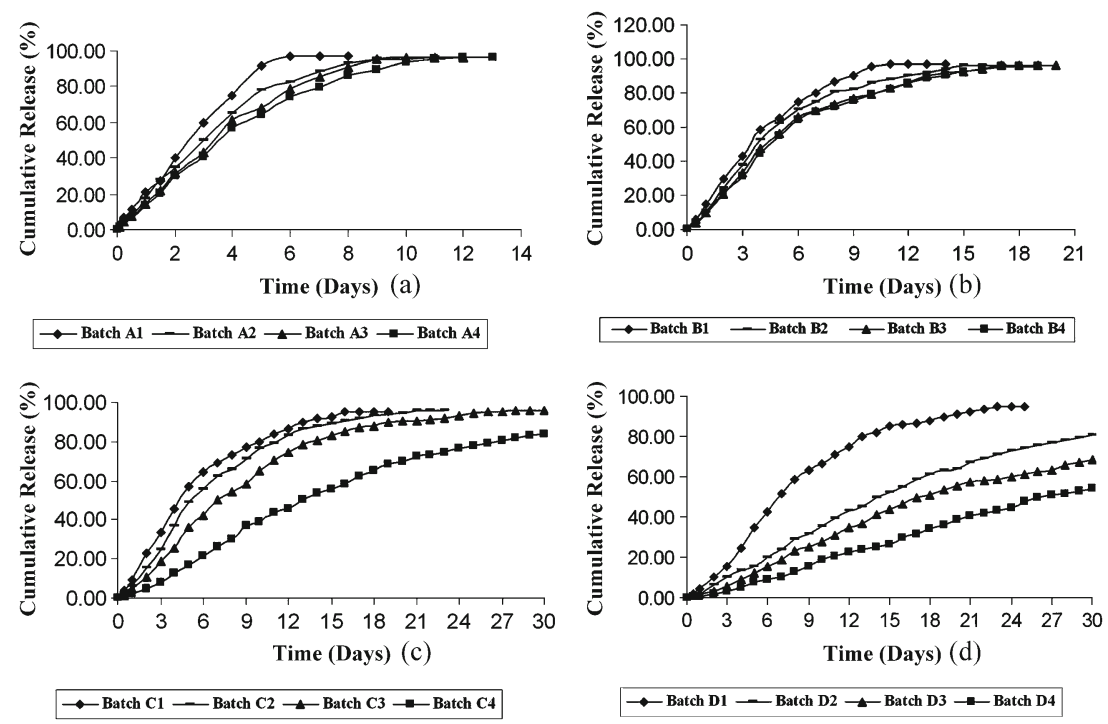

Figure 2. In vitro cumulative release of CTP (\%) over a period of time (in days) for all batches in PBS pH 7.4. (A) for batches $A 1-A 4$, (B) for batches $B 1-B 4$, (C) for batches $C 1-C 4$ and (D) for batches $D 1-D 4$.

Table 2. Results of drug release mechanism showing effect of chitosan concentration and degree of cross-linking calculated from (3) and (4).

\begin{tabular}{|c|c|c|c|c|c|c|c|}
\hline \multirow[b]{2}{*}{ Formulation } & \multicolumn{4}{|c|}{$\begin{array}{l}\text { Power law model } \\
\left(M_{\mathrm{t}} / M_{\mathrm{O}}=k t^{n}\right)\end{array}$} & \multicolumn{3}{|c|}{$\begin{array}{l}\text { Higuchi model } \\
\left(M_{\mathrm{t}} / M=k t^{1 / 2}\right)\end{array}$} \\
\hline & $k$ & $n$ & S.D. & $R$ & $k$ & S.D. & $R$ \\
\hline$A 1$ & $0 \cdot 81$ & $0 \cdot 10$ & $\pm 0 \cdot 031$ & 0.93 & 0.0378 & $\pm 0 \cdot 038$ & 0.97 \\
\hline$A 2$ & $0 \cdot 76$ & $0 \cdot 11$ & $\pm 0 \cdot 024$ & 0.92 & $0 \cdot 0295$ & $\pm 0 \cdot 019$ & 0.95 \\
\hline$A 3$ & 0.74 & $0 \cdot 12$ & $\pm 0 \cdot 008$ & 0.98 & $0 \cdot 0257$ & $\pm 0 \cdot 011$ & 0.99 \\
\hline$A 4$ & 0.74 & $0 \cdot 12$ & \pm 0.009 & 0.99 & $0 \cdot 0252$ & $\pm 0 \cdot 014$ & 0.99 \\
\hline$B 1$ & $0 \cdot 71$ & $0 \cdot 13$ & $\pm 0 \cdot 017$ & 0.96 & $0 \cdot 0238$ & $\pm 0 \cdot 018$ & 0.96 \\
\hline$B 2$ & 0.63 & $0 \cdot 16$ & $\pm 0 \cdot 004$ & 0.99 & $0 \cdot 0181$ & \pm 0.007 & 0.98 \\
\hline$B 3$ & 0.59 & $0 \cdot 18$ & $\pm 0 \cdot 028$ & 0.94 & $0 \cdot 0155$ & $\pm 0 \cdot 023$ & 0.98 \\
\hline$B 4$ & 0.58 & $0 \cdot 18$ & $\pm 0 \cdot 011$ & 0.94 & $0 \cdot 0151$ & $\pm 0 \cdot 023$ & 0.96 \\
\hline$C 1$ & $0 \cdot 61$ & $0 \cdot 17$ & \pm 0.009 & 0.98 & $0 \cdot 0160$ & $\pm 0 \cdot 015$ & 0.96 \\
\hline$C 2$ & $0 \cdot 54$ & $0 \cdot 20$ & $\pm 0 \cdot 009$ & 0.98 & $0 \cdot 0124$ & $\pm 0 \cdot 014$ & 0.99 \\
\hline$C 3$ & 0.45 & 0.23 & $\pm 0 \cdot 015$ & 0.94 & $0 \cdot 0102$ & $\pm 0 \cdot 020$ & 0.97 \\
\hline$C 4$ & $0 \cdot 34$ & $0 \cdot 32$ & $\pm 0 \cdot 018$ & 0.95 & 0.0078 & $\pm 0 \cdot 013$ & 0.97 \\
\hline$D 1$ & $0 \cdot 50$ & $0 \cdot 22$ & $\pm 0 \cdot 006$ & 0.99 & 0.0093 & $\pm 0 \cdot 009$ & 0.98 \\
\hline$D 2$ & 0.41 & $0 \cdot 26$ & $\pm 0 \cdot 013$ & 0.92 & $0 \cdot 0062$ & $\pm 0 \cdot 017$ & 0.96 \\
\hline$D 3$ & $0 \cdot 30$ & 0.35 & $\pm 0 \cdot 016$ & 0.94 & 0.0046 & $\pm 0 \cdot 021$ & 0.98 \\
\hline$D 4$ & $0 \cdot 17$ & 0.52 & $\pm 0 \cdot 013$ & 0.98 & 0.0019 & $\pm 0 \cdot 016$ & 0.99 \\
\hline
\end{tabular}

S.D. stands for standard deviation 
Table 3. Weight loss (\%) of implanted films.

\begin{tabular}{lcccc}
\hline Rabbit no. & $\begin{array}{c}W_{0} \\
(\mathrm{gm})\end{array}$ & $\begin{array}{c}W_{\text {Rem }} \\
(\mathrm{gm})\end{array}$ & $\begin{array}{c}\text { Weight loss } \\
(\%)\end{array}$ & $\begin{array}{c}\text { Mean weight } \\
\text { loss }(\%)\end{array}$ \\
\hline 1. & 0.422 & 0.390 & 7.5 & \\
2. & 0.418 & 0.393 & 6.0 & 7.16 \\
3. & 0.425 & 0.391 & 8.0 & \\
\hline
\end{tabular}

mechanical properties (sufficient strength, water vapour permeation, water absorption capacity and elongation at break for implantation purpose) and desirable in vitro drug release (sustained and nearly complete release within 21-30 days).

\subsection{Kinetic analysis of drug release}

Power law (3) and Higuchi's model (4) are used to analyse the drug release mechanism through the cross-linked $\mathrm{CH}$ films. The drug release through these films depends upon several factors, such as the nature and composition of the films synthesized, cross-link density, physical and mechanical properties of films. Drug release data is plotted in Korsemeyer's equation (3) as log of cumulative percentage of drug released vs $\log$ of time. The values of $n$ and $k$ are determined by applying the linear regression method. The data for release mechanism for different formulations, along with the values of correlation coefficients, ' $R$ ' are presented in table 2 . The values of $k$ and $n$ are varying between 0.17 and 0.81 and $0 \cdot 10$ and $0 \cdot 52$, respectively. The value of $k$ decreases with an increase in concentration of $\mathrm{CH}$ and GL (the crosslinker). On the other hand, the value of diffusional exponent, $n$, increases with an increase in concentration of CH and GL. For all the batches, except batch $D 4$, the value of $n$ is less than $0 \cdot 5$, which implies that the diffusional release mechanism for these batches is quasi-Fickian diffusion; whereas for batch $D 4$, it is non-Fickian.

Higuchi's model (4) describes the release of drug from an insoluble matrix as square root of time-dependent process. The constant, $k$, is calculated from the slope of the plot of cumulative drug released vs the square root of time. The value of $k$ ranges between 0.009 and 0.038 . The value of $k$ decreases with an increase in the concentration of $\mathrm{CH}$ and GL.

So, the kinetic analysis of drug release indicates that the value of $k$ decreases with increase in concentration of polymer and cross-linker used to formulate the films in case of both power level and Higuchi's models; whereas, the value of diffusional exponent, $n$, increases with increase in concentration of polymer and cross-linker. Data indicates that diffusional release mechanism for all the batches, except $D 4$, is quasi-Fickian diffusion; whereas for batch $D 4$, it is non-Fickian.

\subsection{In vivo degradation study}

The $\%$ weight loss of the implanted films over 30 days of implantation in rabbits is reported in table 3 . As only $7 \cdot 16 \%$
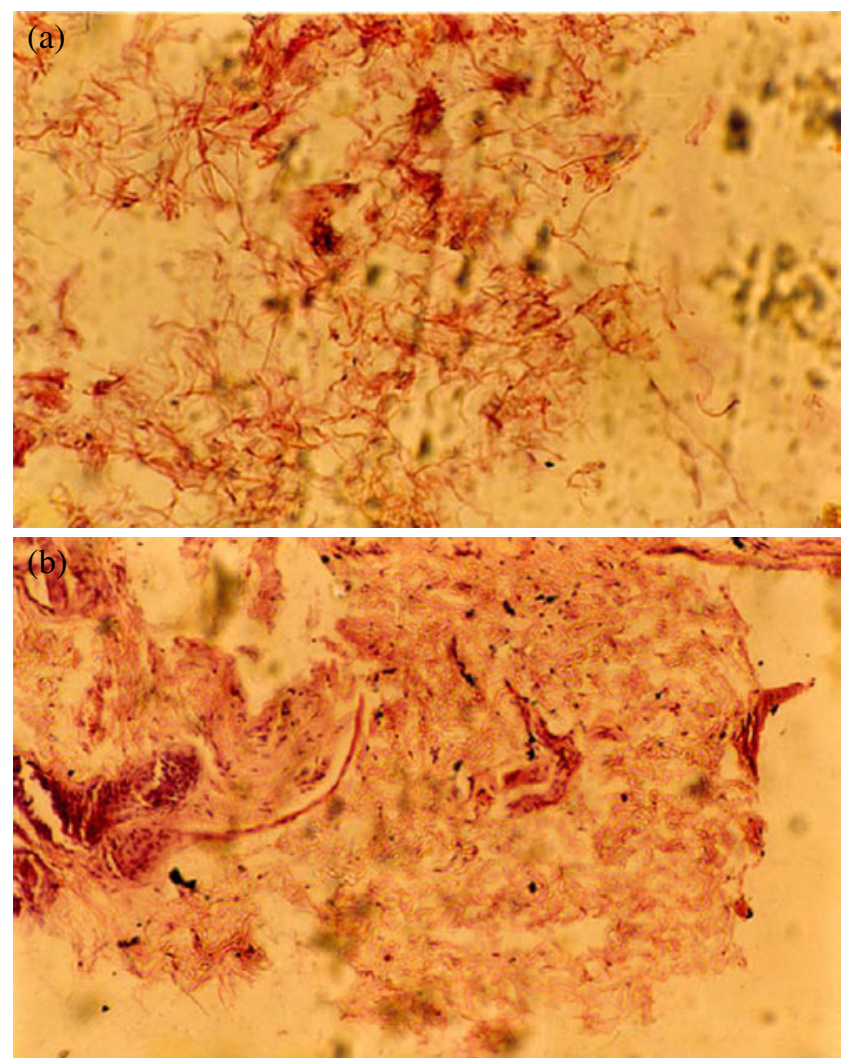

Figure 3. Histopathology slides (a) before and (b) after implantation (magnification, $100 \times$ ) under an optical microscope for histopathological observations of tissue-polymer compatibility in rabbit at thoracic region.

mean weight loss of the implanted film was observed during in vivo degradation study, therefore, the study suggested that batch $C 3$ films could maintain their integrity for desired time period during implantation.

\subsection{Histopathology study}

Histopathology slides of tissues before and after implantation are shown in figures 3 and 4. Histopathology studies of the implants of selected batch films (batch $C 3$ ) for film compatibility showed encouraging results depicting no evidence of any inflammation, any foreign body granuloma or any necrosis or hemorrhage. No changes in tissue configuration are seen before and after 30 days of implantation. 

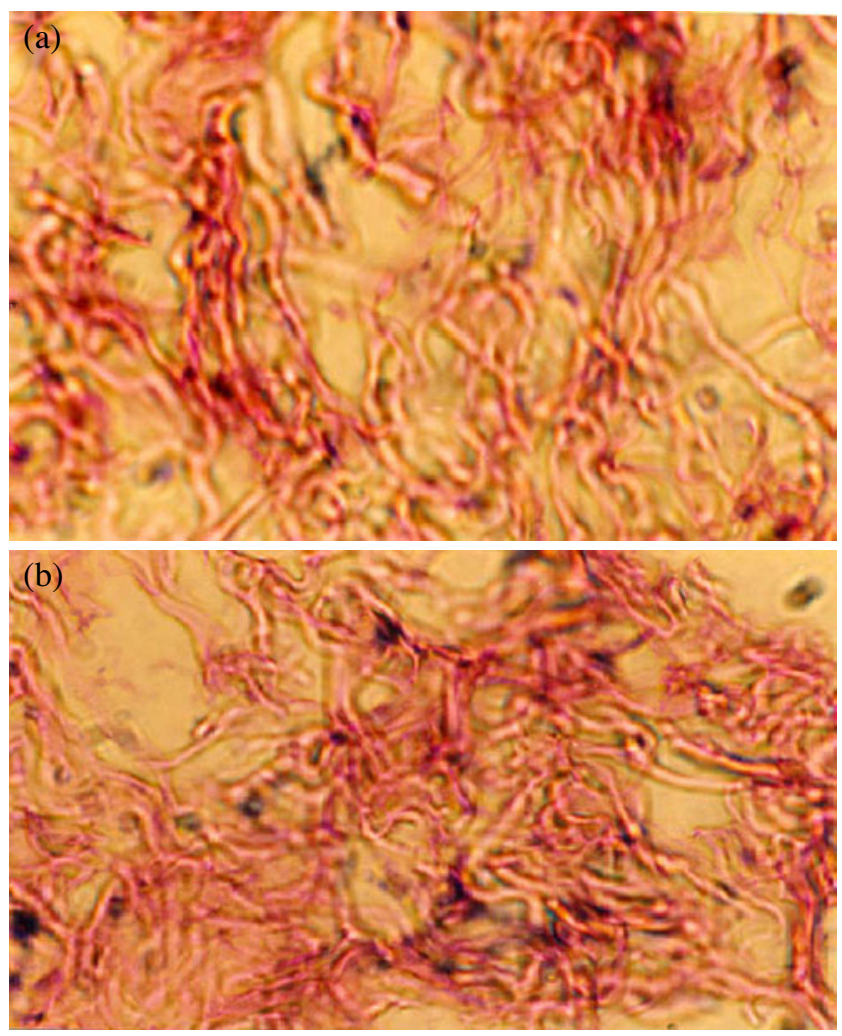

Figure 4. Histopathology slides (a) before and (b) after one month of implantation (magnification $400 \times$ ) under an optical microscope for histopathological observations of tissue-polymer compatibility in rabbit at thoracic region.

Therefore, it can be suggested that the prepared film is compatible and suitable drug carrier for implantation purposes.

\section{Conclusions}

Smooth, clean, firm and tough films are obtained using 1$4 \%$ w/v chitosan, cross-linked with $1-4 \%$ v/v glutaraldehyde. With increase in concentration of $\mathrm{CH}$, water absorption capacity and mechanical strength are increased; whereas water vapour permeability and elongation of the films are decreased. The effect of cross-linking agent, GL, is such that with increase in amount of GL, water vapour permeability, water absorption capacity and elongation of the films are decreased; whereas mechanical strength increased to some extent and then decreased. In vitro release studies indicate that films containing $3 \% \mathrm{CH}$, cross-linked with 2-3\% GL and films containing $4 \% \mathrm{CH}$, cross-linked $1 \% \mathrm{GL}$ are able to sustain the drug release for a prolonged time along with releasing almost complete drug in desired time period. Out of these batches, films containing 3\% $\mathrm{CH}$, cross-linked with 2-3\% GL are having sufficient strength, water vapour permeation, water absorption capacity and elongation at break for implantation purpose. Kinetic analysis of drug release indicates that diffusional release mechanism for all the batches, except $D 4$, is quasi-Fickian diffusion; whereas for batch $D 4$, it is non-
Fickian. The in vitro degradation studies of the films (batch $C 3$ as in table 1) in rabbit indicate that the films maintained their integrity for desired implantation. The histopathological studies of the implanted film ( $C 3$ batch) under microscope indicates that on implanting, there is no evidence of any inflammation, any foreign body granuloma or any necrosis or hemorrhage. Tissue configuration remains unaltered after 30 days of implantation. So, it can be suggested that cross-linked $\mathrm{CH}$ films of above said composition can be used for long term application in depression and related disorders.

\section{References}

Adriano W S, Filho E H C, Silva J A and Goncalves L R B 2005 Biotech. Appl. Biochem. 41201

Andrews J M and Nemeroff C B 1994 Am. J. Med. 9724S

Arvanitoyannis I, Nakayama A and Aiba S 1998 Carbohydr. Polym. 37371

Bayramocglu G, Yylmaz M and Aryca M Y 2003 Biochem. Eng. J. 1335

Beppu M M, Arruda E J, Vieira R S and Santos N N 2004 J. Membr. Sci. 240227

Beppu M M, Vieira R S, Aimoli C G and Santana C C 2007 J. Membr. Sci. 301126

Blanco M D, Gomez C and Olmo R 2000 Int. J. Pharm. 20229

Crini G 2006 Bioresour. Technol. 971061

David P, Manssur Y and Mark S 1992 Carbohydr. Res. 237325

Frazer A 1997 J. Clin. Psychiat. 589

Genta I, Constantini M, Asti A, Conti B and Montanari L 1998 Carbohydr. Polym. 3681

Gliko-Kabir B, Yagen A, Penhasi A and Rubinstein A 2000 J. Control. Rel. 63121

Guibal E 2004 Sep. Purif. Technol. 3843

Gupta K C and Kumar M N V R 2000 Biomaterials 201115

Higuchi T 1963 J. Pharm. Sci. 521145

Jameela S R and Jayakrishnan A 1995 Biomaterials 16769

Jameela S R, Misra A and Jayakrishnan A 1994 J. Biomater. Sci. Polym. Edn. 6621

Jameela S R, Kumary Y V, Lal A V and Jayakrishnan A 1998 J. Control Rel. 5217

Kalapathy U, Proctor A and Shultz J 2000 Bioresour. Technol. 7299 Korsmeyer R W, Gurny R, Doelker E, Buri P and Peppas N A 1983 J. Pharm. Sci. 721189

Miao Y Q and Tan S N 2000 Analyst 1251591

Mi F L, Shyu S S and Wu Y B 2001 Biomaterials 22165

Miyazaki S, Ishii K and Nadai T 1981 Chem. Pharm. Bull. 293067

Muzzarelli R A 1993 Carbohydr. Polym. 207

Ngah W S W, Endud C S and Mayanar R 2002 React. Funct. Polym. 50181

Preskorn S H 1995 J. Clin. Psychiat. 5612

Remunan-Lopez C and Bodmeier R 1997 J. Control. Rel. 44215

Rickels K and Schweizer E 1990 J. Clin. Psychiat. 519

Sezer A G, Hatipoglu F, Cevher E, Oğurtan Z, Bas A L and Akbuğa J 2007 AAPS PharmSciTech. 8 E94

Shu X Z, Zhu K J and Weihong S 2001 Int. J. Pharm. 21219

Thomas C and Sharma P 1990 Biomater. Artif. Cells Artif. Org. 181

Tomihata K and Ikada Y 1997 Biomaterials 18567

Vieira R S and Beppu M M 2005 Adsorption - J. Int. Ads. Soc. 11731

Vieira R S and Beppu M M 2006 Water Res. 401726

Wang L Y, Ma G H and Su Z G 2005 J. Control. Rel. 10662 